\title{
Symptoms of seasonal affective disorder in women diagnosed with early \\ breast cancer receiving radiotherapy
}

William Pidduck MD(C), Bo Angela Wan MD(C), Liying Zhang PhD, Selina Chow BHSc(C), Caitlin Yee BSc(C), Stephanie Chan BSc(C), Leah

Drost BSc(C), Philomena Sousa MBA, Donna Lewis MRT(T), Henry Lam MLS, Eric Leung MD, Edward Chow MBBS

Odette Cancer Centre, Sunnybrook Health Sciences Centre, University of Toronto, Toronto, Ontario, Canada;

MÁnnylrook

\section{Background}

* Seasonal affective disorder (SAD) is characterized by physiological and psychiatric abnormalities in response to changes in seasonal weather patterns and light exposure.

Symptoms typically arise in autumn and winter with remission occurring in the spring and summer months ${ }^{1}$.

Few studies to date have evaluated symptoms of SAD in the context of cancer diagnosis, treatment and survivorship.

\section{Materials and Methods}

Population: Patients at the Sunnybrook Cancer

Center who were diagnosed with non-metastatic breast cancer from 2011-2017 who had >1 Edmonton Symptom Assessment Scale (ESAS) completed preand post-RT

* Available patient databases and chart review yielded data on systemic treatment (chemo/hormone),

radiation, disease characteristics and patient demographics

Patients receiving chemotherapy were not included

Table 2:Comparisons of ESAS Scores before/during/after RT Group A (Spring/Summer) vs. Group B (Winter/Autumn)

\begin{tabular}{|c|c|c|}
\hline & Coefficient* & p-value \\
\hline \multicolumn{3}{|l|}{ Before RT } \\
\hline \multicolumn{3}{|l|}{ Depression } \\
\hline Group A vs. Group B & 0.117 & 0.5755 \\
\hline \multicolumn{3}{|l|}{ Anxiety } \\
\hline Group A vs. Group B & -0.108 & 0.5331 \\
\hline \multicolumn{3}{|l|}{ Overall QOL } \\
\hline Group A vs. Group B & -0.356 & 0.0301 \\
\hline \multicolumn{3}{|l|}{ During RT } \\
\hline \multicolumn{3}{|l|}{ Depression } \\
\hline Group A vs. Group B & -0.144 & 0.6223 \\
\hline \multicolumn{3}{|l|}{ Anxiety } \\
\hline Group A vs. Group B & -0.251 & 0.3625 \\
\hline \multicolumn{3}{|l|}{ Overall QOL } \\
\hline Group A vs. Group B & 0.006 & 0.9747 \\
\hline \multicolumn{3}{|l|}{ After RT } \\
\hline \multicolumn{3}{|l|}{ Depression } \\
\hline Group A vs. Group B & -0.073 & 0.7243 \\
\hline \multicolumn{3}{|l|}{ Anxiety } \\
\hline Group A vs. Group B & -0.097 & 0.5924 \\
\hline \multicolumn{3}{|l|}{ Overall QOL } \\
\hline Group A vs. Group B & -0.013 & 0.9312 \\
\hline
\end{tabular}

*Note: Positive coefficients indicate higher or increased symptom severities with larger magnitudes indicating greater effect sizes.
Results

From total population of 1224 patients this study included: Group A ( $n=84)$ \& Group B ( $n=102)$ (table 1)

Table 1: Timelines for ESAS entry and group inclusion

\begin{tabular}{|c|c|c|}
\hline & $\begin{array}{l}\text { Group A: Spring } \\
\text { and Summer } \\
\qquad \mathbf{N}=\mathbf{8 4}\end{array}$ & $\begin{array}{c}\text { Group B: Autumn and } \\
\text { Winter } \\
\mathrm{N}=102\end{array}$ \\
\hline $\begin{array}{l}\text { Baseline ESAS Entry } \\
\text { (prior to RT) }\end{array}$ & April $1^{\text {st }}$ Aug $31^{\text {st }}$ & Nov $1^{\text {st }}-$ March $15^{\text {th }}$ \\
\hline $1^{\text {st }}$ ESAS Entry post-RT & April $1^{\text {st }}-$ Oct $31^{\text {st }}$ & Nov $1^{\text {st }}-$ May $15^{\text {th }}$ \\
\hline
\end{tabular}

Patients in Group A more likely to have better overall quality of life (QOL) before RT $(p=0.030)$ (Table 2$)$ * Significant differences on absolute score changes (Pre to Post RT) in ESAS depression $(p=0.027)$, anxiety $(p=0.008)$ and overall QOL $(p<0.0001)$ scores between the two groups (Table 3 )

* Group B patients have significantly better improvement on ESAS depression, anxiety and overall QOL scores Pre to Post RT (Table 3)

\section{Discussion/Conclusions}

- Study Limitations

- Retrospective design

- Confounding comorbidities/tx factors

- Small sample size and incomplete data

* Conclusions

- Patients receiving RT in the winter had lower QOL at baseline (pre RT)

- Patients receiving RT in the summer had worse symptom trajectories across all three domains of anxiety, depression and QOL

Table 3: Comparisons of change in ESAS scores between Group A (Summer) vs. Group B (Winter and Autumn)

\begin{tabular}{|l|c|c|} 
& Coefficient* & p-value \\
\hline Depression Changed Score & & \\
\hline Baseline Depression Score & -0.2079 & 0.0178 \\
\hline Group A vs. Group B & 0.5047 & 0.0267 \\
\hline Anxiety Changed Score & & \\
\hline Baseline Anxiety Score & -0.3848 & $<.0001$ \\
\hline Group A vs. Group B & 0.6402 & 0.0079 \\
\hline Overall QOL Changed Score & & \\
\hline Baseline Overall QOL Score & -0.3529 & $<.0001$ \\
\hline Group A vs. Group B & 1.4312 & $<.0001$ \\
\hline
\end{tabular}

